\title{
Use of Ultrasound and Computer Vision for 3D Reconstruction
}

\author{
Ruben Machucho-Cadena ${ }^{1}$, Eduardo Moya-Sánchez ${ }^{1}$, \\ Sergio de la Cruz-Rodríguez ${ }^{2}$, and Eduardo Bayro-Corrochano ${ }^{1}$ \\ 1 CINVESTAV, Unidad Guadalajara, Departamento de Ingeniería Eléctrica y \\ Ciencias de la Computación, Jalisco, México \\ $\{$ rmachuch, emoya, edb\}@gdl . cinvestav . mx \\ 2 Instituto Superior Politécnico "José A. Echeverría", Havana, Cuba \\ sergio@electrica.cujae.edu.cu
}

\begin{abstract}
The main result of this work is an approach for reconstructing the 3D shape and pose of tumors for applications in laparoscopy from stereo endoscopic ultrasound images using Conformal Geometric Algebra. We record simultaneously stereo endoscopic and ultrasonic images and then the 3D pose of the ultrasound probe is calculated using conformal geometric algebra. When the position in 3D of the ultrasound probe is calculated, we compound multiple $2 \mathrm{D}$ ultrasound images into a $3 \mathrm{D}$ volume. To segment $2 \mathrm{D}$ ultrasound images we have used morphological operators and compared its performance versus the obtained with segmentation using level set methods.
\end{abstract}

\section{Introduction}

Endoscopy became an increasing part of daily work in many subspecialties of medicine and the spectrum of applications and devices has grown exponentially [1. The use of a stereoendoscope (i.e. an endoscope with two cameras instead of one) provides more information of the scenario, that is, two slightly different views of the same scene at the same time allows the calculation of the spatial coordinates 2. On the other hand, the ultrasound is found to be a rapid, effective, radiation free, portable and safe imaging modality [3. However, the endoscopic images can not see beyond opaque or occluded structures. The incorporation of ultrasound images into stereoendoscope operative procedures generating more visibility in the occluded regions.

By superimposing Stereo Endoscopic Ultrasound (SEUS) images in the operative field (in the case of laparoscopic or robotic procedures), it would be possible integrate them into a 3D model. This 3D model can help surgeons to better locate some structures such as tumors during the course of the operation. Virtually all available methods use either a magnetic or optic tracking system (and even a combination of both) to locate the tip of the US probe (USP) in 3D space 4. These systems are sometimes difficult to implement in intraoperative scenarios, because the ferromagnetic properties of surgical instruments can affect magnetic tracking systems [5].

E. Bayro-Corrochano and J.-O. Eklundh (Eds.): CIARP 2009, LNCS 5856, pp. 782 789, 2009.

(C) Springer-Verlag Berlin Heidelberg 2009 
We extent a previously proposed method [4] which used just monocular endoscopic images to calculate the pose (i.e. the position and orientation) of the USP. In this paper we use stereo endoscopic images and apply our approach in laparoscopy.

\section{System Setup}

Our experimental setup is illustrated in Figure 1. The equipment setup is as follows: The endoneurosonography equipment (ENS) provides an ultrasound probe (USP) that is connected to an ultrasound system Aloka. The USP is introduced through a channel in the stereo endoscope (Fig. 1a) and is observed by the endoscope.

The USP is flexible and is rotating around the longitudinal axis at about 60 rpm. It can also move back and forth and since the channel is wider than the USP there is also a random movement around the channel. The US image is orthogonal to the USP axis. We know that in small interval of time $\Delta t$, the USP is fixed, and the two endoscopic cameras undergo a movement, which is equivalent to an inverse motion, that is, the endoscopic camera is fixed, and ultrasound probe moves.

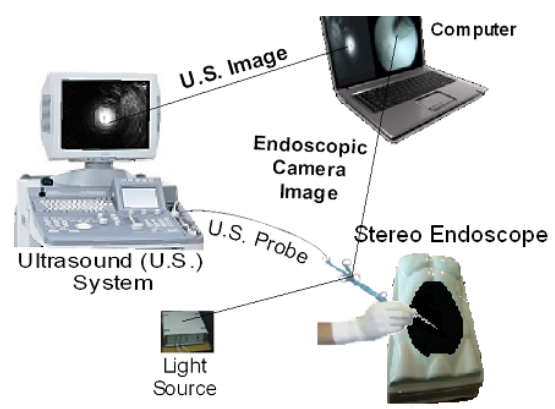

(a)

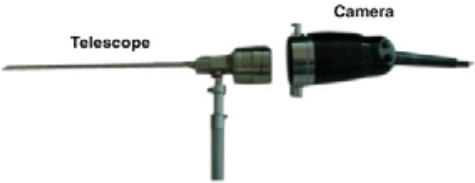

(b)

Fig. 1. a) Experimental setup. b) Equipment, telescope and camera of the stereo endoscope.

\section{Tracking the Ultrasound Probe}

We have used multiple view geometry to process the stereo images; the Figure 2a shows a pair of rectificated stereo images and Fig. $2 \mathrm{~b}$ is its depth map. The cameras were calibrated using the method described in 6]. We track the USP throughout the endoscopic camera images.

In order to track the USP we have used the particle filter and an auxiliary method based on thresholding in the HSV-Space in order to improve the tracking as follows: 


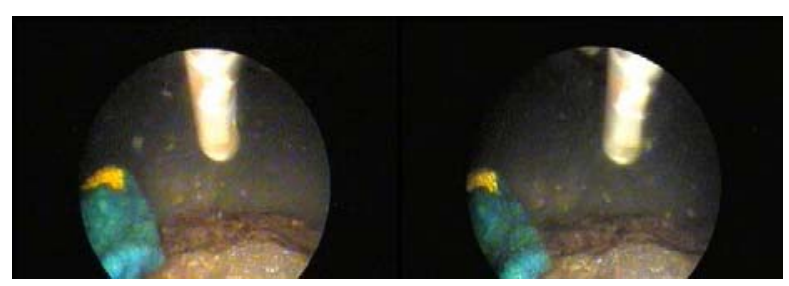

(a)

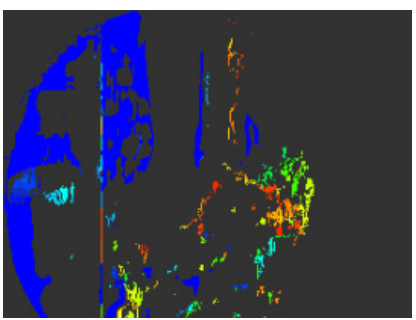

(b)

Fig. 2. a) Pair of rectificated stereo images. b)its depth map.

\subsection{The Particle Filter Tracker}

We used the particle filter to found the USP in the endoscopic images in a similar way to our previous paper [4, with addition of some heuristics. The resultant process is:

- Throw 35 particles on each image and test the likelihood for each of them with respect to a reference histogram. The likelihood is the distance between the color histograms of the model and the current particle. The particle which have the higher likelihood value will be the winner. Then we take the orientation, position and scale from the particle winner as an estimation of the USP in the endoscopic camera image. If the likelihood value of the best particle is higher than a threshold value, we use this information to update the reference model, that is the reference histogram. We have used a threshold value of 0.17 to update the reference model.

- If the likelihood value of the best particle is lower than a threshold value, then we set a flag to enable an additional tracking process in order to find the USP; this additional process is explained in section 3.2. We enable the additional tracking process when the likelihood value is less than 0.08 , otherwise this additional process is disabled.

- We select the 35 particles from a set of particles that we have built for the possible positions, orientations and scales of the USP in the endoscopic image. This set of particles is built off-line and is saved in a text file by using only a chain code for each particle. We also save the size (scale) and the orientation for each particle. In the beginning of the tracking process we read the text file just one time and we store this information in a data structure.

The threshold values and the number of particles (35) were experimentally obtained. Figure 3 a shows the model used to obtain the initial reference histogram. Figure 3 b shows a mask used to build the set of particles. We can see here four points with different colors used as reference in the construction process of the set of particles. The position of the four points is also saved in the text file 
afore-mentioned are used to identify the top of the particle in order to place it correctly on the frame of the endoscopic camera. Figure 3 . shows the used camera frame. It is built just one time at beginning of the tracking process and Figure 4 shows some particles taken from the set of particles. The results of the tracking of the USP are show in Figure 6] we have an example of tracking of the ultrasound probe in the stereo endoscopic camera by using particle filter.

\subsection{Tracking Based on Thresholding in the HSV-Space}

The tracking process is based on thresholding in the HSV-Space and it is used as an auxiliary to find the USP in the endoscopic camera when the likelihood value of the best particle is lower than a threshold value. This works as follows:

- Make a copy of the original image (to preserve it).

- Convert the original image from RGB to HSV-Space.

- Make a binary image by selecting an interval of values from the saturation histogram. This interval should separate the USP from the rest of the image. We have selected the interval $[0,50]$ where we observe the first peak of the histogram.

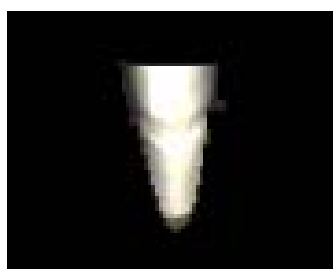

(a)

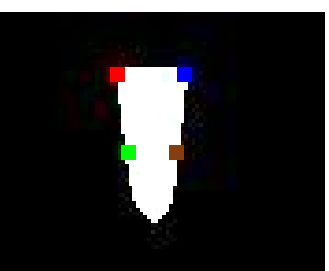

(b)

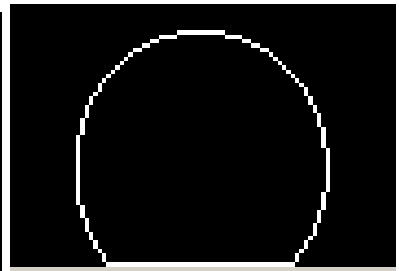

(c)

Fig. 3. a)Model used to obtain the initial reference histogram. b) Model mask used to build the set of particles. c) Camera frame, it is built just one time at the beginning of the tracking process.
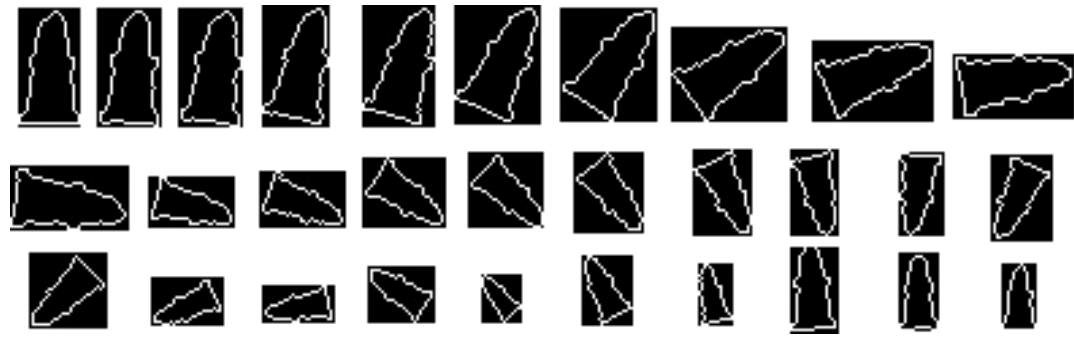

Fig. 4. Set of particles, some particles selected 
Build a new image called sectors, from the binary image and the Canny filter as follows:

- If the pixel $(x, y)$ has an edge (from Canny) and it is part of the mask (binary image) then $(x, y)$ will belong to sectors, see Fig. 5.

- Apply the closing morphological operator to the image sectors in order to fill small holes and to join broken edges. See Fig. 5 f.

- Apply the chain code to calculate the smallest areas of the image sectors, and eliminate them. see Fig. $5 \mathrm{~g}$.

- Get the initial, middle and final of the segmented USP (from the image sectors) on the camera frame and use this information to throw nine particles (replacing the nine particles with the lower likelihood values) in order to improve the tracking process.

Figure 5 illustrates the saturation histogram for the endoscopic camera image shown in Fig. 5b. Figure 50 shows its saturation image and Figure 5] is the application of the Canny filter to the original image. This tracking method is just used as a support method because it does not take into account temporal information and because is also sensible to partial occlusions of the USP in the endoscopic cameras images as well as background, illumination and contrast variations.

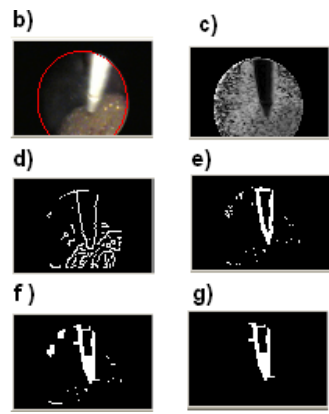

Fig. 5. Tracking process in the HSV-Space
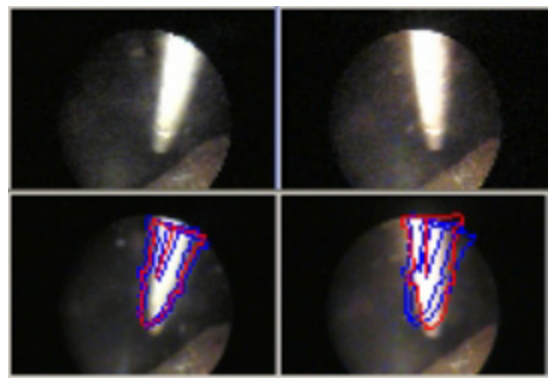

Fig. 6. Example of Tracking of the USP. The best particle is shown in red color. 


\section{Ultrasound Image Processing}

We have used two methods in order to process the ultrasound images; the first one is based in morphological operators 4 and the second one is the level sets method.

The level sets method uses an initial seed on the image. This seed evolves with the time until a zero velocity is reached or the curve is collapsed (or a maximum number of iterations is reached). To evolve the curve, the method uses two lists called Lin and Lout 7 . We present the results of the processing and a comparison between both methods. They work independently of the tumor characteristics.

Figure 7 shows the results obtained by using morphological operators. Figure $7 \mathrm{a}$ is an original ultrasound image. In Figure $7 \mathrm{~b}$ the central part of the image is excluded, because it only contains noise and the ROI is selected. The binary mask obtained for this method that will be applied to the original image is showed in Figure 7f and Figure 7 $\mathrm{d}$ shows the result of the segmentation.

Figure 8 shows the results obtained by using the level sets method. Figure $8 \mathrm{a}$ is an original ultrasound image. Figure $8 \mathrm{c}$ shows the ROI selected. Figure $8 \mathrm{~d}$ shows the initial seed applied to Figure 8 r. Figure 8 e shows the collapsed curve. Figure 8f is the binary mask obtained from Figure 83. This mask is applied to the original image and so we have obtained the result of the segmentation (Figure 8b. Both figures were obtained from the Aloka ultrasound equipment by using a box shaped rubber phantom.

\subsection{Comparison between Both Methods}

We have obtained a processing time of 0.005305 seconds for the morphological operators method versus 0.009 seconds for the level sets method, that is $188 \mathrm{fps}$ versus $111 \mathrm{fps}$. We recommend both methods for in line implementation, because they are fast and reliable.

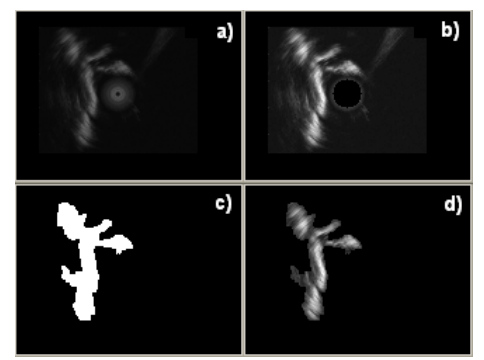

Fig. 7. Isolating the tumor. a) Original US image to be segmented. b) The central part of the image is excluded. c) ROI. d) Result of segmentation. 


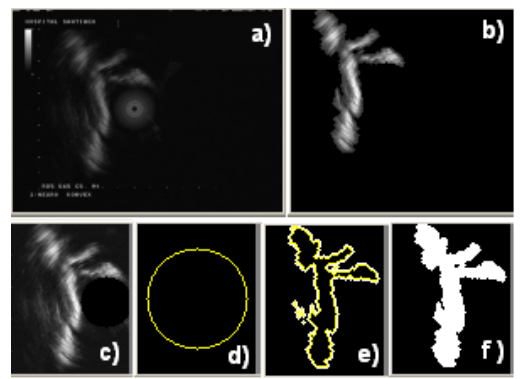

Fig. 8. Segmentation using the level sets method

\section{Calculating the 3D Pose of the Tumor}

This work make use Conformal Geometric Algebra (CGA) to represent geometric entities ( points, lines, planes, spheres, etc.) in a compact and powerful form [8]. The CGA preserves the Euclidean metric and adds two basis vectors: $\mathrm{e}_{+}, \mathrm{e}_{-}$ (where $\mathrm{e}_{+}{ }^{2}=1$ and $\mathrm{e}_{-}{ }^{2}=-1$ ), which are used to define the point at the origin $\mathrm{e}_{0}=\frac{1}{2}\left(\mathrm{e}_{-}-\mathrm{e}_{+}\right)$and the point at the infinite $\mathrm{e}=\mathrm{e}_{-}+\mathrm{e}_{+}$. The points in CGA are related with the Euclidean space by: $\underline{p}=\mathbf{p}+\frac{\mathbf{p}^{2}}{2} \mathrm{e}+\mathrm{e}_{0}$. A sphere in dual form is represented as the wedge of four conformal points that lies on sphere $\underline{s}^{*}=\underline{a} \wedge \underline{b} \wedge \underline{c} \wedge \underline{d}$, its radius $\rho$ and its center $\underline{p}$ in $\mathcal{R}^{3}$ can be obtained using: $\rho^{2}=\frac{\underline{s}^{2}}{(\underline{s} \cdot e)^{2}}, \underline{p}=\frac{\underline{s}}{-(\underline{s} \cdot e)}+\frac{1}{2} \rho^{2}$ e. A plane in dual form is defined as a sphere, but the last point is at the infinity: $\underline{\pi}^{*}=\underline{a} \wedge \underline{b} \wedge \underline{c} \wedge$ e. A line in dual form is represented as the wedge of two points and the infinity point: $\underline{L}^{*}=\underline{a} \wedge \underline{b} \wedge$ e. A line can also be calculated as the intersection of two planes: $\underline{L}=\pi_{1} \wedge \bar{\pi}_{2}$. This equation is used to calculate the $3 \mathrm{D}$ line that represents the ultrasound probe axis. To achieve a translation by a distance $d_{2}$ from a point $p_{1}$ in the direction of a line and to obtain $\underline{p_{2}}: T=\exp \left(\frac{1}{2} d_{2} L\right), \underline{p_{2}}=T \underline{p_{1}} \widetilde{T}$. The last equation is used to find the position of the ultrasound sensor in order to put the segmented ultrasound image in $3 \mathrm{D}$ space; where $p_{1}, p_{2}$ represent the begin and the end respectively of the best particle on the stereo endoscopic images and $d_{2}$ is the retroprojected distance between them.

\subsection{Results}

Figure 9] shows a convex hull applied to a set of slices of tumor in the 3D space and, Figure 9b shows the part of the phantom used and the tumor.

\section{Conclusions}

We have addressed the problem of obtaining 3D information from joint stereo endoscopic and ultrasound images obtained with SEUS equipment. We have used 


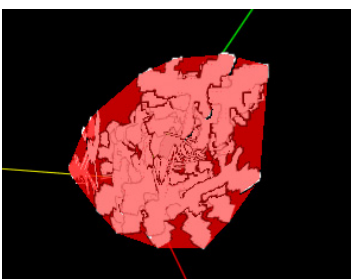

(a)

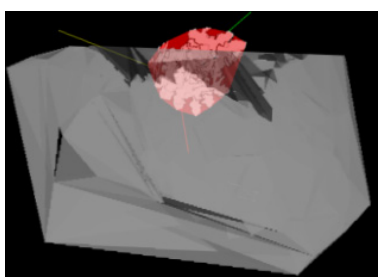

(b)

Fig. 9. 3D Reconstruction from a set of slices of tumor

conformal geometric algebra to calculate the pose of the ultrasound sensor in order to put the segmented tumor in the 3D space. To verify the segmentation of the tumor in the ultrasound images, we have compared two different segmentation methods and obtained good results at a reasonable speed for both. Some preliminary results are presented.

\section{References}

1. Muller, U.: Possibilities and limitations of current stereo-endoscopy. Surg. Endosc 18, 942-947 (2004)

2. Senemaud, J.: 3D-Tracking of minimal invasive instruments with a stereoendoscope. $\mathrm{PhD}$ thesis, Universität Karlsruhe, Karlsruhe, Germany (2008)

3. Nascimentoa, R.G., Jonathan, C., Solomon, S.B.: Current and future imaging for urologic interventions. Current Opinion in Urology 18, 116-121 (2008)

4. Machucho Cadena, R., de la Cruz Rodriguez, S., Bayro Corrochano, E.: Joint freehand ultrasound and endoscopic reconstruction of brain tumors, pp. 691-698 (2008)

5. Hummel, J.B., et al.: Design and application of an assessment protocol for electromagnetic tracking systems. Medical Physics 32, 2371-2379 (2005)

6. Bayro Corrochano, E., Daniilidis, K.: The dual quaternion approach to hand-eye calibration. In: International Conference on Pattern Recognition, vol. 1 (1996)

7. Shi, Y.: Object-based Dynamic Imaging with Level Set Methods. PhD thesis, Boston University, Boston, U.S.A. (2005)

8. Bayro Corrochano, E.: Geometric Computing for Perception Action Systems: Concepts, Algorithms, and Scientific Applications. Springer, Heidelberg (2001) 